DujIC, Dejan

DOI: 10.15170/DIKE.2018.02.02.08

Ehem. Obersekretär des Rotkreuzausschusses

PTE ÁJK

\title{
Der Gedanke eines Mannes, „Menschen zu helfen“ wird zur größten humanitären Organisation der Welt
}

\begin{abstract}
The Idea of a Man "to Help People" Becomes the Largest Humanitarian Organization in the World

This article deals with the emergence of the largest international humanitarian movement and its structure. In other words, how Jean Henry Dunant's idea of helping people in need, became a bumanitarian organization in a minimum of time, that spread across all five continents and performs its activities, protected by international law and the Geneva Conventions 1-4, for more than 150 years. The structure of the Red Cross and Red Crescent organization is based on three partly structurally, independent elements: the International Red Cross and Red Crescent societies, the International Federation of Red Cross and Red Crescent Societies and the International Committee of the Red Cross.
\end{abstract}

Keywords: Jean Henry Dunant, humanitarian organization, Red Cross, Red Crescent, non-governmental organization, Geneva Conventions, military law

\section{Einführung}

Die Geschichte der Menschheit begleiten seit jeher Kriege und die damit in Zusammenhang zu bringenden verheerenden Massen an verletzten und Menschenopfern. Schon in der frühen Zeitgeschichte stellte man sich zwar die Frage, was mit den verletzen Soldaten und Kriegshinterbliebenen geschehen soll, jedoch gab es bis in das späte 19. Jahrhundert kein institutionell organisiertes international-einheitliches Übereinkommen über die Verpflegung der im Krieg verletzten Soldaten. ${ }^{1}$

Natürlich gab es zwar eine Art Verpflegung; einige Heere hatten sogenannte Feldlazarette oder sogar Ärzte. Bekannt ist auch, dass manche Länder gegenseitig Abkommen geschlossen haben über die beidseitige Verpflegung und Versorgung aller im Krieg verletzten Soldaten und der Bevölkerung (1759, Frankreich - England). Es wurden, zumindest nach den heute noch zur Verfügung stehenden Aufzeichnungen, zwischen 1581 und 1864 insgesamt sogar 291 Übereinkommen über Versorgung und Verpflegung der Kriegsverletzten getroffen, jedoch fehlten Standards, allgemein gültige Verfahren, institutionell und international aufgestellte verbindliche Normen und sogenannte Durchführungsorgane für die Verletzten. ${ }^{2}$ Wenn es auch Versorgungen

\footnotetext{
${ }^{1}$ Hantos, A Magyar Vöröskereszt 100 éve 19.

${ }^{2}$ MVK-OI, Magyar Szent Korona Országai Vőrős-Kereszt Egyletének története 2-6.
} 
gab, lag dies eher im Ermessen der Heeresführung und größtenteils an der Bereitwilligkeit der einheimischen Bevölkerung, da diese ihre Hilfe, wenn überhaupt, nur freiwillig angeboten hat. Es gab ja keinen entsprechenden Paragrafen, der die unterlassene Hilfeleistung in Strafe gestellt hätte.

\section{Von der Schlacht in Solferino bis zu den ersten Schritten}

Das Verlangen der Bevölkerung nach einer internationalen Regelung, näher einer Regelung auf Völkerrechtsebene bezüglich der Versorgung, Verpflegung und Behandlung der im Krieg verwundeten Soldaten und der betroffenen Bevölkerung, wurde immer stärker. Diese Gedanken wurden auch durch die in Frankreich zu dieser Zeit stattfindenden Revolution und die Ideen der Aufklärung immer mehr geschürt, jedoch musste fast ein Jahrhundert vergehen, bis sich aus diesen Bestrebungen eine - damals noch als Hilfsorganisation bezeichnete - Organisation entwickelt.

1859 fand in Solferino die - wie es zeitgeschichtliche Quellen oft bezeichnen - grausamste Schlacht des Jahrhunderts zwischen den napoleonischen und den österreichisch-kaiserlichen Streitkräften statt. Jean Henry Dunant $(1828-1910)^{3}$, der sich dort mit Napoleon III wegen Bankgeschäften treffen wollte (er sollte ihm finanzielle Mittel zur Errichtung einer Mühle beisteuern), sah auf dem Schlachtfeld die dort stattfindenden Grausamkeiten. Man muss beachten, dass in der Schlacht von Solferino immerhin 40.000 Soldaten ums Leben gekommen und verletzt worden sind, und viele von ihnen haben keine fachgerechte Versorgung bekommen. Der Gestank und die qualvollen Leidesschreie haben Dunant zutiefst getroffen.

Jedoch sah er auch das gute. Die Dorfbewohner von Solferino haben sich zusammengetan und haben als freiwillige Helfer, so gut es ging, die Kranken und Verletzten versorgt. Diese uneigennützige freiwillige Hilfestellung war in dieser Zeit nicht selbstverständlich oder gängig, jedoch bestärkte es Dunant in dem Gedanken, dass eine internationale Regelung und eine internationale (unabhängige) Hilfsorganisation vonnöten ist, die unparteiisch allen hilft, die den Kriegen zum Opfer fallen, egal aus welchen Gründen dies auch geschieht. ${ }^{4}$ Die sich ihm in Solferino gebotenen Eindrücke ließen den damals noch jungen Dunant nicht mehr ruhen. Er war im Zwiespalt. Einerseits bereitete ihm das Gemetzel von Solferino schlaflose Nächte und versetzte ihn in Angst, andererseits hat ihn die aufopferungsvolle Hilfe der Bewohner von Solferino fasziniert. Er fand es beeindruckend, wie den Betroffenen ohne beachten ihres Offiziersgrades, ihrer Herkunft geholfen wurde. Alleine entscheidend war, so hat es zumindest Dunant empfunden, ob und wie schwer die betroffenen Personen verwundet sind. Sonst wurde jeder absolut gleichbehandelt, wie es später auch eines der Grundprinzipien des International Committee of the Red Cross und somit auch der nationalen Rotkreuz- und Rothalbmondgesellschaften wurde.

\footnotetext{
${ }^{3}$ Jean Hennry Dunant ist am 8. Mai 1828 in Genf geboren. Er stammt aus einer Wohlhabenden Unternehmerfamilie. Schon in jungen Jahren war er sehr sozial angehaucht und wirkte in mehreren Hilfsorganisationen mit. Sein Leben nahm jedoch eine tragische wende, mit 39 ist er - da er alle seine finanziellen Mittel und Kraft in das Rote Kreuz gesteckt hatte - mittellos geworden. Erst 1895, nachdem ein schweizerischer Journalist mit ihm ein Interview gemacht hat, wurde der Gesellschaft klar, er lebt noch. 1897 wurde Dunant vom Bundesrat der Binet-Fendt-Preis verliehen, 1901 erhielt er gemeinsam mit dem französischen Pazifisten Frédéric Passy den Friedensnobelpreis. Seine letzten Jahre verbrachte er in einer Obdachlosenunterkunft, die das Rote Kreuz leitete, wo er am 30. Oktober 1910 verstarb. FRANÇOIS, Un grand humanitaire 203-244.

${ }^{4}$ MVK-OI, Vöröskereszt története Eszmeterjesztőknek 20-25.
} 


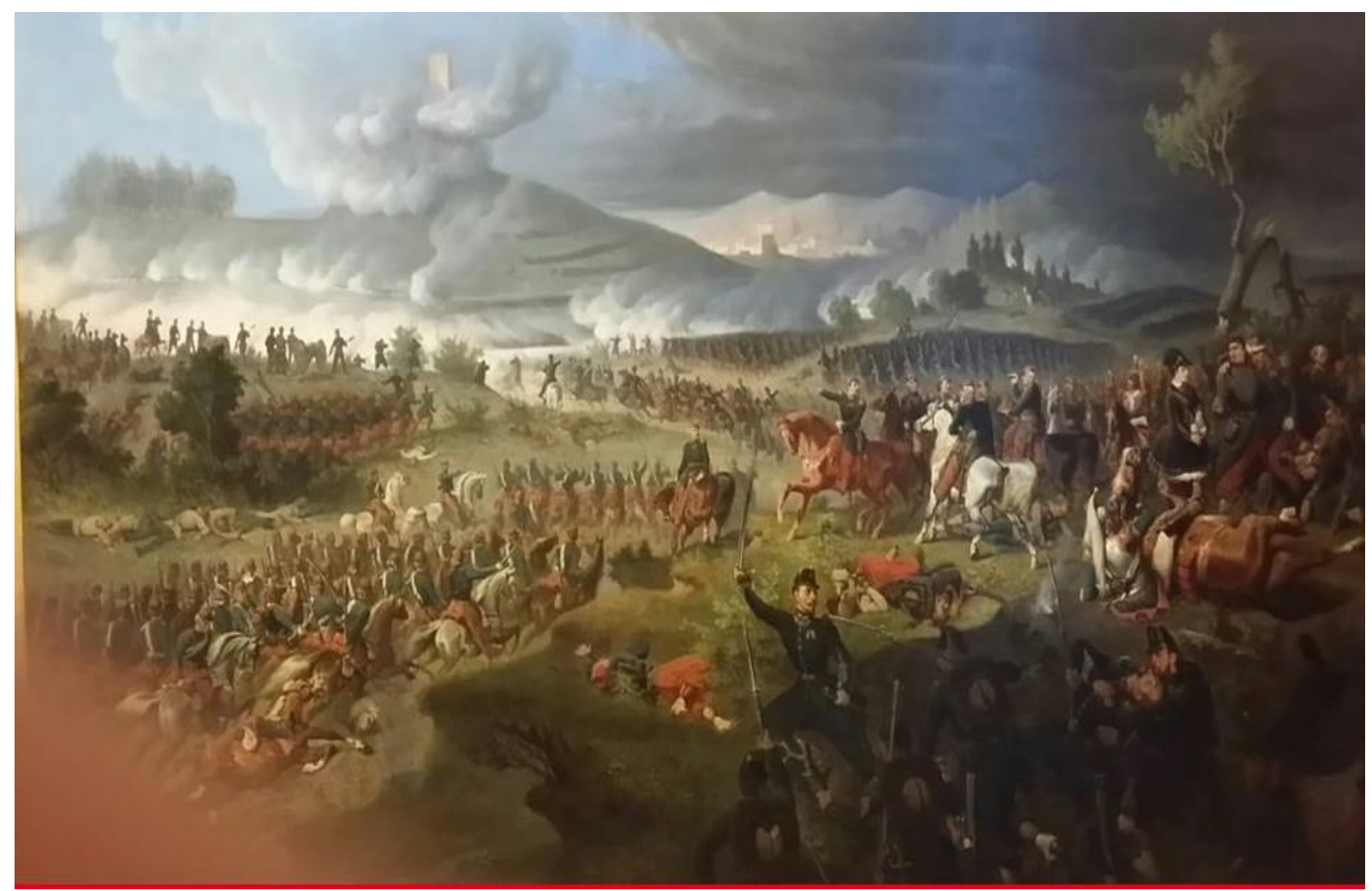

1. Bild: Horace Vernet: Die Schlacht von Solferino. Vernet Muzeum Versailles (Benutzt bei Vorlesungen des Roten Kreuzes)

Dunant konnte nicht anders, er entschied sich seine Eindrücke auf Papier zu bringen. Sein Plan war es, den Herrschern und den Entscheidungsträgern der damaligen Zeit die Grausamkeiten und die Folgen eines Krieges bildlich darzustellen und sie aufzurütteln: auch während und nach einem Krieg müssten die Regeln eingehalten werden und man dürfe die Menschen, die sich für ein Land aufopfern, nicht einfach vergessen und ihrem eigenen Schicksal überlassen. Sein Buch Un souvenir de Solferino (Eine Erinnerung an Solferino, 1862), gilt heute noch als literarisches Meisterstück, mit dem er die damalige Gesellschaft in Europa aufrüttelte. In seinem Buch schilderte Dunant zunächst die politischen Zusammenhänge, und informierte detailliert über das Militär und Kriegsstrategien. ${ }^{6}$

Die letzten Seiten widmete Dunant seiner Vision: „Wäre es nicht möglich, in Friedenszeiten eine freiwillige Organisation zu gründen, deren Zweck es sein müsste, die Verwundeten in Kriegszeiten durch begeisterte und aufopfernde Freinillige, die für ein solches Werk besonders geeignet sind, pflegen zu lassen?" Er schloss sein Buch mit dem Appell: „Hilfsgesellschaften für Verwundete sollten in verscbiedenen Ländern Europas gegründet werden ".7 Dunant ließ auf eigene Rechnung 1.600 Exemplare drucken, die er mit persönlicher Widmung an Europas Herrscherfamilien, militärische Würdenträger, Generäle und Regierungen in ganz Europa verschickte. Der Erfolg des Buches war gewaltig. Eine zweite Auflage erschien schon

\footnotetext{
${ }^{5}$ Das Buch erschien in ungarischer Übersetzung als eine Eigenausgabe des Ungarischen Roten Kreuzes 1978.

${ }^{6}$ https://www.drk.de/das-drk/geschichte/wissen-und-helfen/biografie-henry-dunant/eine-erinnerung-an-solferino/

${ }^{7}$ DUNANT, Solferinoi emlékek 41-45.
} 
vier Monate nach der ersten und wurde zum Bestseller. Dunant wurde Weltberühmt, man behandelte ihn wie einen Star. Der Erfolg seiner Idee war so gut wie sicher.

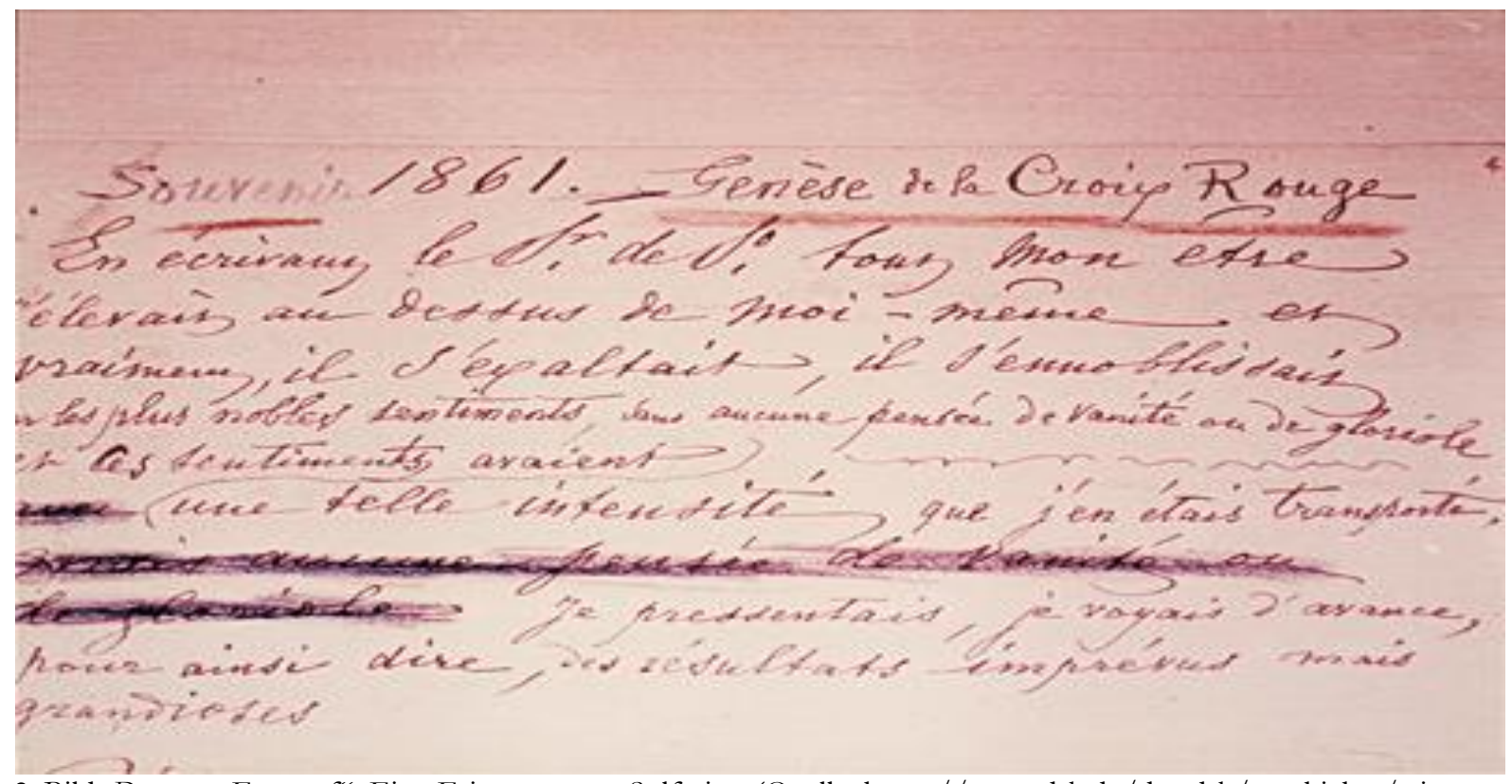

2. Bild: Dunants „Entwurf“. Eine Erinnerung an Solferino (Quelle: https://www.drk.de/das-drk/geschichte/wissenund-helfen/biografie-henry-dunant/eine-erinnerung-an-solferino/)

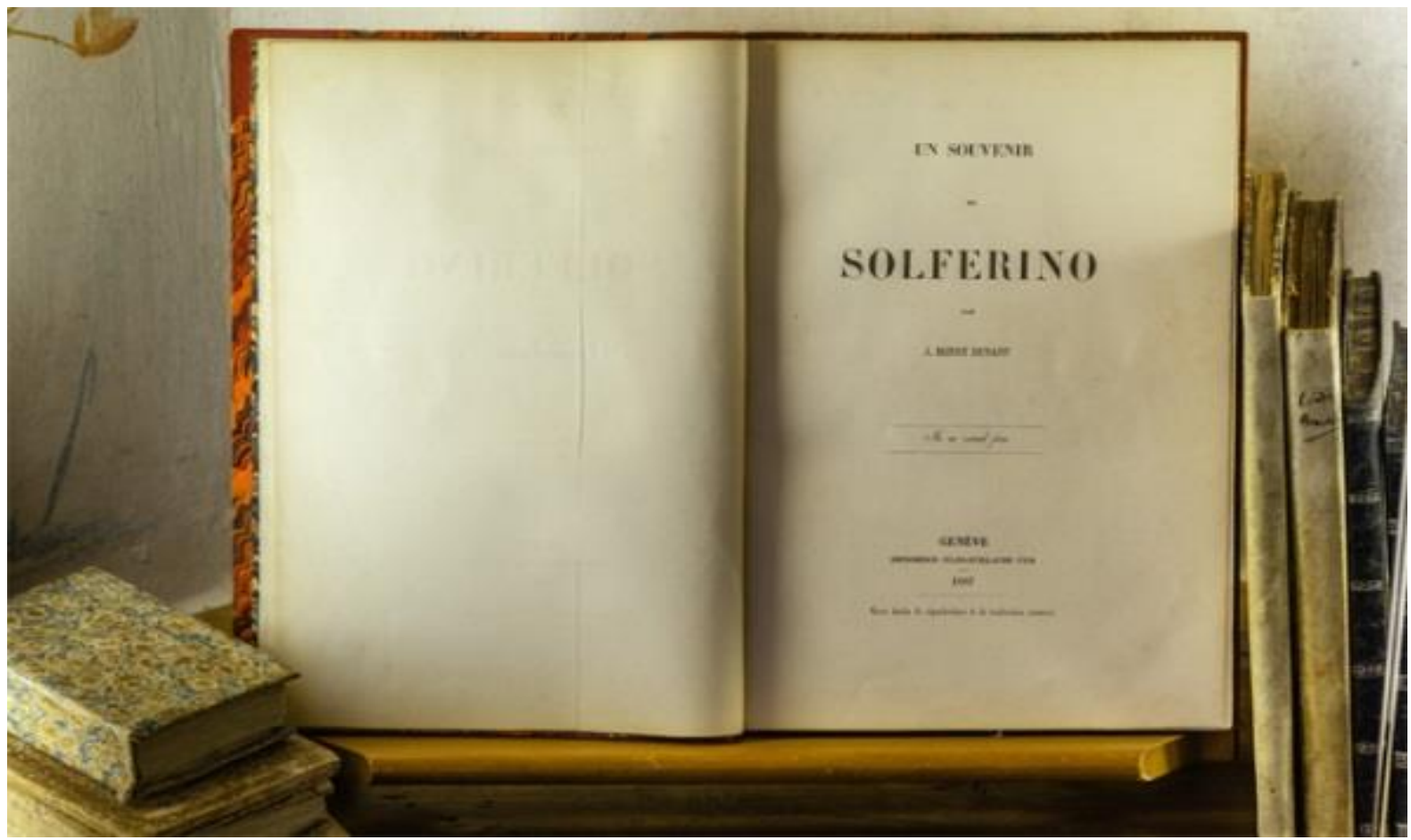

3. Bild: Buchtitel - Eine Erinnerung an Solferino (Quelle: https://www.drk.de/das-drk/geschichte/wissen-undhelfen/biografie-henry-dunant/eine-erinnerung-an-solferino/) 
Einer der Ersten, die ihm gratulierten, war der Genfer Anwalt Gustave Moynier, ein hervorragender Jurist und erprobter Organisator. Er bot und sicherte Dunant seine Hilfe und Unterstützung zu. Er konnte Dunant für ein Referat vor zwanzig angesehenen Bürgern der Stadt gewinnen, unter ihnen General Guillaume Henri Dufour. ${ }^{9}$

Das Schlusswort des Buches von Dunant diente quasi als Auftakt seines Referates und als Grundstein für die Gründung des sogenannten Internationalen Komitees der fünf Vorsitzenden. Durch seinen sehr emotionalen Vortrag und seines gut gelungenen Buches konnte er also fünf wichtige Persönlichkeiten für seine Idee begeistern: „(...) Gibt es während einer Zeit der Rube und des Friedens kein Mittel, um Hilfsorganisationen zu gründen, deren Ziel es sein müsste, die Verwundeten in Kriegszeiten durch begeisterte, aufopfernde Freiwillige, die für ein solches Werk besonders geeignet sind, pflegen zu lassen? (...) Da man nun einmal darauf verzichten muss, dass sich Wünsche und Hoffnungen der Gesellschaft der Friedensfreunde jemals erfüllen werden, warum sollte man da nicht eine Zeit verbältnismässiger Rube und Stille benutzen, um eine Frage von so grosser und umfassender Wichtigkeit von dem doppelten Standpunkt der Menschlichkeit und des Christentums aus zu studieren? Warum sollte man nicht versuchen, hierüber zu einem Entschluss zu kommen? (...) Gesellschaften solcher Art würden, sobald sie einmal für die Dauer errichtet sind, natürlich zu Friedenszeiten untätig bleiben, aber sie würden in ständiger Bereitschaft sein für den Fall eines Krieges. (...) Hätte es bei Solferino ein solches internationales Hilfswerk gegeben, oder wären am 24., 25. und 26. Juni in Castiglione oder zur gleichen Zeit auch in Brescia, Mantua oder Verona solche freiwilligen Helfer gewesen, wieviel unbeschreiblich Gutes bätten sie leisten können in jener unheilvollen Nacht vom Freitag zum Samstag, als Tausende von Vervundeten vor Qual stöhnten und herzzerreissend um Hilfe riefen, Tausende, die nicht nur unter furchtbaren Schmerzen, sondern auch unter einem entsetzlichen Durst litten. (...) Wäre es nicht wünschenswert, dass die hohen Generäle verschiedener Nationen, wenn sie gelegentlich zusammentreffen, diese Art von Kongress dazu benützen, irgendeine internationale rechtsverbindliche und allgemein hochgehaltene Übereinkunft zu treffen, die, wenn sie erst festgelegt und unterzeichnet ist, als Grundlage dienen könnte zur Gründung von Hilfsgesellschaften für Verwundete in den verschiedenen Ländern Europas? (...). ‘ 10

Moynier, der neben seinen anwaltlichen Tätigkeiten auch Vorsitzender der in Genf ansässigen Privaten Gemeinnütrigen Gesellschaft Genfs (Societe Genevoise d'Utilite Publique) war, fand Dunants vorgetragene Idee des neutralen und unabhängigen Sanitätsdienstes so genial, dass er Ihm anbot, durch die Unterstützung und die Mithilfe seiner Gesellschaft die Idee von Dunant aufzugreifen und es zu ermöglichen seinen Vorschlag, dass in Friedenszeiten freiwillige Helfer ausgebildet werden, deren Neutralität und Unabhängigkeit auch auf dem Schlachtfeld greift mit Unterstützung und der Mithilfe des Rates der Privaten Gemeinnütrigen Gesellschaft Genfs durchzusetzen. ${ }^{11}$ Näher die Errichtung eines dauerhaften Sanitätsdienstes, der in Kriegszeiten der Armee zur Seite gestellt wird um neutral, fachgerecht und ohne unnötig zwischen den verletzten zu unterscheiden, die Verpflegung und Versorgung der Verletzten gewährleistet.

\footnotetext{
${ }^{8}$ Gustave Moynier (1826 - 1910), der hervorragender Genfer Jurist, war Vorsitzender der I. Genfer Konferenz (1863). Er war es auch, der den Entwurf für das erste Genfer Abkommen verfasst hatte. Siehe DURAND, Gustave Moynier and the peace societies.

9 General Guillaume Henri Dufour (1787 - 1875), Obergeneral der Schweizer Streitmächte. Seines Verhandlungsgeschickes ist es zu verdanken, dass der 12 andauernde Sonderbundkonflikt 1847 friedlich gelöst werden konnte. Siehe PETERS, Transitions in Engineering.

${ }^{10}$ DUNANT, Eine Erinnerung an Solferino 40-105.

${ }^{11}$ Hantos, A Magyar Vöröskereszt 100 éve 24.
} 
Der Rat entschloss für die gründliche Untersuchung des Themas ein Komitee einzurichten, das sich damit befassen soll, die Möglichkeiten für die Realisierung der Vorschläge von Dunant zu erarbeiten und die organisatorischen Aufgaben einzuleiten. Somit entstand am 9. Februar 1863 das sogenannte Internationale Komitee der fünf Vorsitzenden, dessen Mitglieder Dufour, Dunant, Moynier sowie zwei Ärzte, Théodore Maunoir ${ }^{12}$ und Louis Paul Amédée Appia ${ }^{13}$ waren. Dieses Komitees wurde den Vorreiter des künftigen Internationalen Komitees der Rotkreuz- und Rothalbmondgesellschaft.

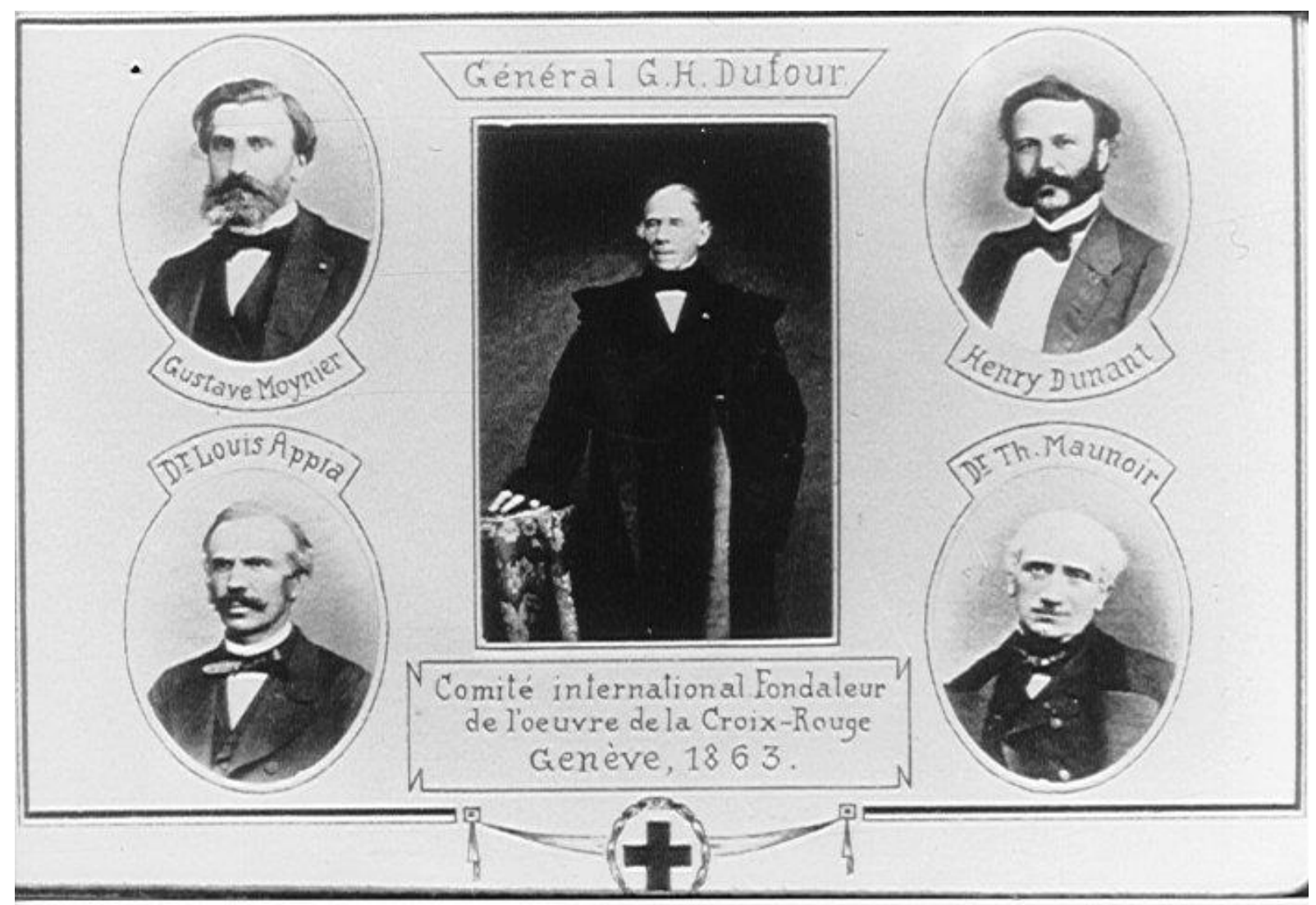

4. Bild: Zeitgenössische Darstellung der fünf Gründungsmitglieder aus dem Archiv des Ungarischen Roten Kreuzes

Im Namen des Komitees nahm Dunant im September 1863 als Gesandter an dem internationalen Kongress für Statistik in Berlin teil, wo er verkündete, dass am 26. Oktober 1863 in Genf eine internationale Konferenz mit dem Titel Das Erricbten dauerbafter und internationaler Hilfsorganisationen zur Hilfeleistung und Hilfestellung für die im Krieg verwundeten Soldaten stattfinden wird. ${ }^{14}$ Seine Verkündung kam bei den Teilnehmern des Kongresses sehr gut an, 14 Länder

12 Théodore Maunoir (1807 - 1869) war Chirurg in Paris und in Genf. Siehe DuRAND, Théodore M. est aussi un fondateur de la Croix-Rouge 139-155.

${ }^{13}$ Louis Paul Amédée Appia (1818-1898) war als freiwilliger Chirurg an der Seite der Französisch-Sardischen Truppen als Feldarzt tätig. Siehe BOPPE, L'homme et la guerre. Le Docteur Louis Appia et les débuts de la Croix-Rouge.

${ }^{14}$ Hantos, A Magyar Vöröskereszt 100 éve 24. 
(Unteranderem das Land Baden, Hessen-Darmstadt, Preußen, Italien und Spanien) sagten zu, an der Konferenz teilzunehmen und Vertreter zu schicken, die im Namen der Regierung vollumfängliche und rechtskräftige Übereinkommen treffen und gegebenenfalls einem internationalen Abkommen zustimmen können. $\mathrm{Zu}$ den Beschlüssen, geprägt durch die Vorstellungen und die Forderungen Dunants, die er den Vertretern der 14 Länder vorgetragen hat, zählten unter anderem die folgenden: 1. die Gründung nationaler Hilfsgesellschaften in Europa für Kriegsverwundete; 2, die neutrale Behandlung der Verwundeten; 3, die Entsendung freiwilliger und bereitwilliger Pflegekräfte so wie Helfer zur Unterstützung und Hilfestellung auf den betroffenen Schlachtfeldern; 4, die Organisation und Durchführung weiterer Treffen als auch internationaler Konferenzen; 5, die Einführung einer einheitlichen Kennung zum Schutz der freiwilligen Helfer in Form eines roten Kreuzes auf weißem Hintergrund. Diese Zeichen war das Inverse zur schweizerischen Flagge, das bewusst so gewählt wurde um die Neutralität und Unabhängigkeit der Organisationen zu unterstreichen.

Somit konnte Dunant mit Stolz behaupten, dass die zentralen Fragen, die er in seinem Buch Eine Erinnerung an Solferino behandelte und gestellte, entsprechend berücksichtigt wurden. Diese Konferenz wurde auch als Gründungsmoment des Internationalen Rotkreuz und Rothalbmond deklariert. Während dieser Konferenz wurde auch die Gründungsurkunde des nunmehr international fungierenden Roten Kreuzes unterzeichnet, und binnen paar Monaten erreichte das internationale Komitee, dass sich 14 Staaten am 29. Oktober 1863 der Idee und dem Komitee anschlossen. Ein weiterer Meilenstein und als Erfolg dieser Konferenz ist zu verzeichnen, dass die Konferenz zugleich den Grundstein für die I. Genfer Konvention gelegt hatte.

\section{Die Ergebnisse der Genfer Konferenz und ihre Folgen}

Dem Erfolg der Genfer Konferenz verdankend, hat die schweizerische und französische Regierung am 8. August 1869 zur Erlassung der I. Genfer Konvention eine diplomatische Versammlung einberufen. Es wurden alle europäischen Länder sowie die Vereinigten Staaten von Amerika, Mexiko und Brasilien eingeladen. An der diplomatischen Konferenz nahmen 16 Länder mit insgesamt 26 Delegierten teil. In der I. Genfer Konvention betreffend die Linderung des Loses der im Felddienst verwundeten Militärpersonen, die seit ihrem Entstehen (22. August 1864) als Grundcharta erst dem Internationalen Komitee der Hilfsgesellschaften für Verwundetenpflege, dann seinem Rechtsnachfolger, dem International Committee of the Red Cross (ICRC) dient, ist eine der wichtigsten völkerrechtlichen Übereinkommen: Der Schutz des Sanitätswesens im Kriege wurde festgelegt weiterhin bestimmt, dass das Personal der Lazarette, Verbandsplätze und Medizindepots als neutral anzusehen sind, solange sie ihrer Funktion nachgehen. Ein rotes Kreuz auf weißem Grund wird als Schutzzeichen festgelegt. ${ }^{15}$ Artikel 1 legte grundlegend fest, dass „,alle Militärpersonen und andere den Heeren dienstlich beigegebene Personen, die verwundet oder krank sind, obne Unterschied der Staatsangehörigkeit von der Kriegspartei, in deren Händen sie sich befinden, geachtet und versorgt werden sollen " 16

\footnotetext{
15 https://www.welt.de/geschichte/article167862925/Die-erste-Genfer-Konvention-wird-unterzeichnet.html

16 I. Genfer Konvention (auch Abkommen genannt) in der Originalfassung. Seit dem wurden die vier Genfer Konventionen und ihre Zusatzprotokolle mehrfach geändert. Der Aktuelle Stand der Konventionen basiert auf der Überarbeitung von 1949.
} 
Diese I. Genfer Konvention war in ihrer ursprünglichen Fassung das erste völkerrechtliche Dokument zum Schutz der Neutralen und der sich freiwillig aufopfernden Helfer. Sie wurde am 22. August 1864 nach langen Gesprächen und einigen Modifikationen von 22 Ländern unterschrieben. Die rechtsgeschichtliche Bedeutung erlangt die I. Genfer Konvention darin, dass sie als erste verbindliche Völkerrechtsnorm nunmehr die Länder, die sich dieser Konvention anschlossen, zur Einhaltung der entsprechenden Vorschriften verpflichtete. Seine erste richtige Bewehrungsprobe hatte die Konvention 1870 im Krieg zwischen Preußen und Frankreich, da dies beides Staaten die Konvention ratifizierten. Fraglich war, ob sich die zwei Streitkräfte an die Bestimmungen der Konvention halten, ob die aus 12 Ländern gesandten medizinischen Fachkräfte und das neutrale Hilfspersonal seine Arbeit unversehrt und uneingeschränkt auf beiden Seiten verrichten können. Auch wenn ein Krieg nie als etwas Positives anzusehen ist, konnte festgehalten werden, dass die Konvention ein voller Erfolg war, und seine Ziele erreicht hatte. ${ }^{17}$

Von dem Erfolg beflügelt wollte die nun aufblühende internationale Rotkreuz- und Rothalbmond-Organisation mehr erreichen. Es folgten - basierend auf der I. Genfer Konvention - weitere völkerrechtliche Normen. Am 06. Juli 1906 wurde die II. Genfer Konvention verabschiedet mit dem Titel zur Verbesserung des Loses der Verwundeten, Kranken und Schiffbrüchigen der Streitkräfte zur See. Nach dem I. Weltkrieg standen die Länder und die Weltbevölkerung erneut vor neuen Herausforderungen. Die Gefangenen des I. Weltkrieges wurden grausam behandelt, unmenschlichen Qualen ausgesetzt. Die Würde des Menschen wurde missachtet, somit musste eine weitere Norm auf Völkerrechtsebene geschaffen werden, um diese Missstände einigermaßen in den Griff zu bekommen. 1929 wurde die III. Genfer Konvention erlassen mit dem Titel Abkommen über die Behandlung der Kriegsgefangenen, dem die IV. Genfer Konvention zum Schutz der Zivilpersonen in Kriegszeiten 1949 folgte. Die vier Konventionen in ihrer neuen Fassung von 1949 dienen bis heute mit ihren beiden Zusatzprotokollen von 1977 als Kernstücke des humanitären Völkerrechts. Die Genfer Konventionen traten am 21. Oktober 1950 in Kraft, die Zusatzprotokolle am 7. Dezember 1978. Bis 2002 haben 190 Staaten die vier Genfer Konventionen, 160 Staaten das I. Zusatzprotokoll und 153 Staaten das II. Zusatzprotokoll ratifiziert, und ermöglichen dadurch das geschützte, neutrale und unabhängige Arbeiten der Rotkreuz- und Rothalbmondgesellschaften. ${ }^{18}$

\section{Die nationalen und internationalen Gesellschaften}

Natürlich kann man sich die Frage stellen, warum dieser Abschnitt der Abhandlung mit den nationalen Rotkreuz-, Rothalbmond- und Rotkristallgesellschaften anfängt, da es eindeutig unmöglich ist alle Gesellschaften im Detail zu behandeln, und diese - zumindest könnte man das glauben - mit dem internationalen Roten Kreuz und Roten Halbmond nichts zu tun haben. Richtig ist aber diese Herangehensweise allemal, da genau diese nationalen Gesellschaften den Grundstein für das International Committee of the Red Cross (ICRC) und den International Federation of Red Cross and Red Crescent Societies (IFRC) geben, ferner werden nicht die einzelnen landesspezifischen Züge

\footnotetext{
${ }^{17}$ Hantos, A Magyar Vöröskereszt 100 éve 30.

${ }^{18}$ Eine jeweils aktuelle Liste findet sich auf der Website des ICRC (www.icrc.org/eng/party_gc)
} 
dargestellt, sondern die allgemeinen und für alle nationalen Gesellschaften verbindlichen Regularien.

Ohne die nationalen Gesellschaften könnten die internationalen Organisationen nicht existieren, sie wären nichts mehr als leere Begriffe. Die nationalen Gesellschaften der internationalen Organisation führen die tatsächlichen Aufgaben des ICRC und der IFRC aus. Sie sind im weiteren Sinne die ausführende Gewalt, abhängig davon, ob die internationale Organisation ihre Tätigkeit in der Friedenszeit (IFRC) oder im Kriegszustand/Katastrophengebieten (ICRC) ausübt. Dies Bedeutet aber nicht, dass die zwei tatsächlichen Organisationen kein Personal oder Aufgaben hätten. Das ICRC spielt, auch für die nationalen Organisationen eine zentrale Rolle, die IFRC in der technischen- infrastrukturellen Ausrichtung im Fuhrparkwesen und in der Luftfahrt. Die ersten nationalen Gesellschaften wurden nach der Genfer Konferenz (26. Oktober 1863) gegründet, nachdem die teilnehmenden 14 Staaten die Vorschläge von Dunant angenommen und ratifiziert hatten. In den folgenden Jahren schlossen sich immer mehr Staaten der - damals noch unter dem Namen Internationales Komitee der Hilfsgesellschaften für Verwundetenpflege bekannten Organisation an. Nach diesem Erfolg hat das internationale Komitee eine Reihe an Maßnahmen ergriffen um den Eintritt der nationalen Gesellschaften zu koordinieren. Es wurden Grundregel und Prinzipien und Voraussetzungen ausgearbeitet, die eine nationale Gesellschaft und der Staat, in dem sich diese etablieren möchte, erfüllen müssen um in die Organisation aufgenommen zu werden. Es wurden auch die bis heute geltenden sieben Grundsätze erarbeitet, an die sich jede nationale Gesellschaft während seiner Tätigkeit zu halten hat. das Unterzeichnen der Gründungsurkunde drückt es aus, dass sie sich diesen unterstellt werden. Diese Prinzipien sind wie folgt.

1. Die Menschlichkeit: „Die internationale Rotkreuz- und Rothalbmond-Bewegung, entstanden aus dem Willen, den Verwundeten der Schlachtfelder unterschiedslos Hilfe zu leisten, bemüht sich in ibrer internationalen und nationalen Tätigkeit, menschliches Leiden überall und jederzeit zu verbüten und zu lindern. Sie ist bestrebt, Leben und Gesundheit zu schützen und der Würde des Menschen Achtung zu verschaffen. Sie fördert gegenseitiges Verständnis, Freundschaft, Zusammenarbeit und einen dauerhaften Frieden unter allen Völkern".

2. Die Unparteilichkeit: „Die Rotkren₹- und Rothalbmond-Bewegung unterscheidet nicht nach Nationalität, Rasse, Religion, sozialer Stellung oder politischer Überzengung. Sie ist einzig bemüht, den Menschen nach dem Maß ibrer Not zu helfen und dabei den dringendsten Fällen den Vorrang zu geben ".

3. Die Neutralität: „Um sich das Vertranen aller zu bewabren, enthält sich die Rotkreuq- und Rothalbmond-Bewegung der Teilnahme an Feindseligkeiten wie auch, zu jeder Zeit, an politischen, rassischen, religiösen oder ideologischen Auseinandersetzungen".

4. Die Unabhängigkeit: „Die Rotkreuz- und Rothalbmond-Bewegung ist unabhängig. Wenn auch die nationalen Gesellschaften den Bebörden bei ibrer bumanitären Tätigkeit als Hilfsgesellschaften zur Seite stehen und den jewviligen Landesgesetzen unterworfen sind, müssen sie dennoch eine Eigenständigkeit bewabren, die ihnen gestattet, jederzeit nach den Grundsätzen der Rotkreuz- und Rothalbmond-Bewegung zu handeln".

5. Die Freiwilligkeit: „Die Rotkreu₹- und Rothalbmond-Bewegung verkörpert freiwillige und uneigennützige Hilfe obne jedes Gewinnstreben". 
6. Die Einheit: „In jedem Land kann es nur eine einzige nationale Rotkreu₹- oder RothalbmondGesellschaft geben. Sie muss allen offenstehen und ibre humanitäre Tätigkeit im ganzen Gebiet ausüben".

7. Die Universalität: „Die Rotkreu₹- und Rothalbmond-Bewegung ist weltumfassend. In ibr haben alle nationalen Gesellschaften gleiche Rechte und die Pflicht, einander zu belfen". 19

Neben den sieben Grundsätzen muss das neue Mitglied (der Mitgliedsstaat und die nationale Gesellschaft) dem allgemeinen Verfahren vor dem in Genf ansässigen Ausschusses der Organisation, das ein Organ des internationalen Rotkreuz- und Rothalbmond-Bewegung ist, standhalten. Demzufolge muss die neu zugründende nationale Gesellschaft und der Staat, wo die neue Gesellschaft gegründet werden soll die Grundcharta, also die I. Genfer Konvention anerkennen und dieser beitreten. Für alle, die für die Rotkreuz- und Rothalbmond Organisationen tätig sind (egal ob nationale Gesellschaft, Haupt oder Ehrenamt, dauerhaft oder nur aushilfsweise), gelten die sieben Grundsätze, sowohl die daraus resultierenden Rechte als auch die daraus folgenden Pflichten. Darum spricht die Grundsatzcharta der internationalen Organisation von einer Bewegung. Das unterstreicht die Einheitlichkeit derer aller, die für die Organisation tätig sind oder in ihrem Namen Aufgaben ausüben.

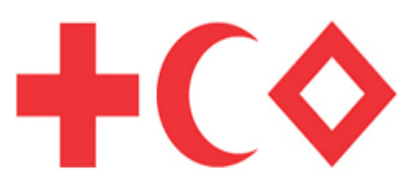

5. Bild: Die drei offiziellen Symbole der nationalen Gesellschaften

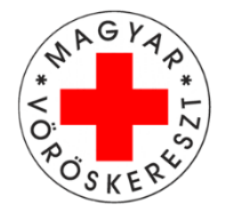

6. Bild: Das nationale Symbol des Ungarischen Roten Kreuzes

In den Konferenzen von Genf zwischen 1863 und 1864 haben sich die Länder und das Internationale Komitee darauf geeinigt, dass alle sich der Bewegung anschließenden nationalen Gesellschaften, alle Gebäude, Flug- und Fahrzeuge einheitliche Symbole benutzen werden um allen sichtbar zu machen, dass hier das unabhängige, neutrale und unter dem Schutz des humanitären Völkerrechts stehende internationale und nationale Hilfskomitee seine Tätigkeit ausübt von dem, für keinen eine Gefahr ausgeht oder besteht. Ursprünglich hatte man die Inverse der Schweizer Flagge (roter Hintergrund weißes Kreuz - weißer Hintergrund Rotes Kreuz) als gemeinsames Symbol festgelegt, jedoch war es problematisch dieses Zeichen in muslimischen Ländern zu verwenden. Vor allem das damals noch osmanische Reich (bis 29. Oktober 1923) und andere muslimischen Länder beharrte darauf, dass neben dem Roten Kreuz auf weißem Hintergrund auch der Rote Halbmond aufgenommen werden soll. Somit wurde, als das osmanische Reich 1876 dem

19 Jede Nationale Gesellschaft hat diese Grundsätze in Handbuch und Unterrichtsformat in der jeweiligen Landessprache zur Hand. Siehe z. B. Deutsches Rotes Kreuz: Das Rote Kreuz und seine Grundsätze. https://www.drk.de/das-drk/geschichte/wissen-und-helfen/das-rote-kreuz/die-sieben-rotkreuzgrundsaetze/ 
Internationalen Komitee beitrat, auch der Rote Halbmond auf weißem Hintergrund aufgenommen. Bis zum 8. Dezember 2005 gab es auch kein anderes Symbol, jedoch um den Eintritt von Israel zu erleichtern wurde vom ICRC das sogenannte Symbol des dritten Protokolls, umgangssprachlich der Rote Kristall mit weißem Hintergrund unter die Offiziellen Schutz und Hinweissymbole aufgenommen. Sofern die nationalen Gesellschaften ihre Tätigkeiten im Inland ausüben (Jugendschutz, Blutspendendienst, Rettungsdienst, Leitung von Krankenhäusern und Wohneinrichtungen usw.), ist um das rote Symbol herum immer die Bezeichnung der jeweiligen nationalen Gesellschaft zu lesen bzw. bekommen auch die Gebäude weiterhin die Flug- und Fahrzeuge das Symbol. ${ }^{20}$

Der Rotkreuz- und Rothalbmond-Bewegung (der Kristall wird auch nach 2005 im Namen der Bewegung nicht erwähnt), gehören 185 nationale Gesellschaften an (Stand 2006). Das Rote Kreuz benutzen 145, den Roten Halbmond 35 und den Roten Kristall 1 nationale Gesellschaft. Somit kann man erkennen, dass die Bewegung sich auf alle Kontinente erstreckt und das von den anerkannten unabhängigen Ländern der Welt ${ }^{21} 185$ Länder eine nationale Gesellschaft gegründet haben und somit der I. Genfer Konvention auch angehören. Das Anerkennen der Genfer Konvention bedeutet jedoch nicht automatisch, dass auch eine nationale Rotkreuz- oder Rothalbmondgesellschaft gegründet werden muss, ist aber die Voraussetzung der Gründung. ${ }^{22}$

Die Aufgabe einer jeden nationalen Gesellschaft ist auch die medizinische, soziale Hilfestellung, die Leitung und Errichtung sozialer, medizinischer, Jugend- und Senioreneinrichtungen, des Weiteren die Sicherstellung der Blutreserven und der notfallmedizinischen Versorgung (Rettungsdienst, Katastrophenschutz) in dem Land, in dem die Gesellschaft gegründet wurde. In diesem Sinne ist sie nur national (innerländlich) tätig, jedoch ist eine jede nationale Gesellschaft verpflichtet auf Anweisung und unter der Koordination und Leitung des ICRC in Zusammenarbeit mit anderen nationalen Gesellschaften und dem ICRC auch international aufzutreten. In dem Land, wo das ICRC als Leiter und Koordinator seine Tätigkeiten ausübt, hat es die Rechte und Befugnisse für alle Aufgabenausübungen, die der Rotkreuz- und Rothalbmondbewegung völkerrechtlich eingeräumt worden sind. Somit übernimmt in dem Zeitraum das ICRC auch die nationalen Tätigkeiten, und die betroffene Gesellschaft schmilzt während dieser Zeit mit den anderen dort zur Hilfestellung gekommenen nationalen Gesellschaften in die strukturelle Organisation des ICRC ein. Dieser absolut logische Schritt und die Vorgehensweise des ICRC, alle mitwirkende Gesellschaften in diesem Zeitraum einzugliedern, hat seine Daseinsberechtigung darin, dass das ICRC in erster Linie seine Tätigkeiten in Kriegsgebieten und außerordentlich hervorstechenden Katastrophengebieten ausübt, daraus resultierend das nötige Knowhow und die infrastrukturellen Möglichkeiten besitzt die Situation fachgerecht zu handhaben.

\footnotetext{
${ }^{20}$ MVK-OI, Vöröskereszt története eszmeterjesztőknek 25-78.

${ }^{21}$ Nach dem Abkommen der Vereinten Nationen 194, de jure 204 Länder der Welt.

22 MVK-OI, Vöröskereszt története eszmeterjesztőknek 25-78.
} 


\section{Das International Committee of the Red Cross und seine Stellung als Akteur des Völkerrechts}

In der schon erwähnten Dreierstruktur (die nationale Gesellschaft, das ICRC, das im deutschen Sprachraum auch Internationale Komitee des Roten Kreuzes genannt ist, und der IFRC) ist das einzige und originäre Völkerrechtsakteur der Bewegung. Das ICRC ist die Organisation, die das tatsächliche internationale Rote Kreuz darstellt. Diese Organisation ist es, die damals 1863 als das Komitee der 5 gegründet worden ist, dann als Internationales Komitee der Hilfsgesellschaften für $V$ ervundetenpflege bezeichnet seine Tätigkeiten aufnahm. Wenn man heute darüber spricht, so auch die Quellen, wird die Gründung des ICRC also auf den 17. Februar 1863 zurückdatiert (Gründung des Internationalen Komitees). Die jedoch heute benutzte Bezeichnung ICRC entstand erst um 1876 nach dem Eintritt des osmanischen Reiches.

Näher betrachtet bedeutet dies, dass das ICRC neben den nationalen Gesellschaften eine eigenständige juristische Persönlichkeit besitzt, des Weiteren neben dem Heiligen Stuhl des Papstes und der Malteser Hilfsorganisation die einzige nicht staatliche, den Staaten untergeordnete und von denen abhängige Organisation ist, die völkerrechtliche Rechtsfähigkeit besitzt, in ihrem eigenen Namen und für die gesamte Bewegung durch völkerrechtliche Verträge, Verhandlungen und Abkommen, Rechte zu erlangen und Pflichten nachzukommen. Da das ICRC nicht den Staaten der Welt untergeordnet ist und nicht im engen Sinne von Staaten gegründet wurde (diese nur durch die Anerkennung der I. Genfer Konvention den Vorgaben des ICRC zustimmen), gilt im Sinne des Völkerrechts das ICRC als non-governmental organisation (genannt auch NGO), die jedoch völkerrechtlich den von den Vereinten Nationen anerkannten Staaten der Welt gleichzustellen ist.

Ihren Sitz hat das ICRC in Genf, besitzt eine eigene Organisationsstruktur und umfasst eine Mitarbeiterzahl von ca. 20.000 (teils direkt Beschäftigte bei dem ICRC, teils Mitarbeiter, die auch für die einzelnen nationalen Gesellschaften angestellt sind). Das ICRC hat neben dem Sitz um die 100 weiteren strategisch wichtigen Stützpunkte u. a. in Budapest oder Wien. Die Aufgaben des ICRC sind mannigfaltig, von der Aufnahme und Überwachung der nationalen Gesellschaften bis hin zur Mitwirkung bei der Gestaltung des humanitären Völkerrechtes. Sie leitet auch eine Vielzahl von humanitären Operationen, stellt Schiffe, Flugzeuge technische Hilfsmittel zur Verfügung, wirkt in der Versorgung der Flüchtlinge auf der ganzen Welt mit und hilft den nationalen Staaten bei der Bewältigung allerlei Katastrophen, Hungersnöten und anderen nicht vorhersehbaren vis maior Zuständen, die die nationalen Gesellschaften alleine nicht bewältigen können. ${ }^{23}$ In erster Linie beschränkt sich die Tätigkeit des ICRC auf Aufgaben, die mit Kriegszuständen, innerstaatlich zustande kommenden, durch Waffengewalt unterstützten Aufständen oder deren Folgen zusammenhängen. Zur Stärkung und das unterstützen der nationalen Gesellschaften in ihrer Arbeit, sofern diese die Arbeit in Friedenszeiten ausüben, wurde 1919 die Föderation gegründet.

${ }^{23}$ MVK-OI, Vöröskereszt története Eszmeterjesztőknek 78-105. 


\section{Der International Federation of Red Cross and Red Crescent Societies}

Nach dem I. Weltkrieg haben die Siegermächte das ICRC aufgesucht und Verhandlungen angestrebt. Ihr Vorschlag war, dass das Internationale Komitee des Roten Kreuzes durch die Stärkung der nationalen Gesellschaften dazu beiträgt, dass die Bewegung auch in Friedenszeiten mehr Aufgaben übernimmt. Begründung war, dass die meisten vom Komitee ausgeübten Tätigkeiten auch in der Friedenszeit für die Bevölkerung von Bedeutung sind und die Bevölkerung oft auch in Friedenszeiten in Not geraten kann. Mit Zustimmung des ICRC wurde daraufhin in Zusammenarbeit der Siegermächte des I. Weltkrieges mit dem Namen die Liga des Internationalen Roten Kreuzes, des Roten Halbmondes und des Roten Löwen die zweite internationale Organisation der Bewegung gegründet. Grund für die Aufnahme des Roten Löwen war der Eintritt von Iran 1922 in die internationale Bewegung. Da das damals noch unter dem Einfluss des persischen Kaiserreichs und der kaiserlichen Familie war, bestand Iran darauf der kaiserlichen Tradition entsprechend, dass die Liga auch den Roten Löwen als Symbol der Liga aufnimmt. Nach der Revolution und der politischen Umstrukturierung Irans (1980) wurde der Rote Löwe als Symbol entfernt. Iran benutzt von da an auch den Roten Halbmond. ${ }^{24}$

Die Hauptaufgaben der Föderation liegen in erster Linie in der Stärkung und der Unterstützung der nationalen Gesellschaften. Konkret ist die Föderation nichts anders als das Gesamtbündnis aller nationalen Gesellschaften, somit sind die Aufgaben der Föderation dieselben als die der nationalen Gesellschaften. Der Unterschied liegt darin, dass sobald sich mehrere nationale Gesellschaften zusammenschließen und im Ausland tätig werden sie als die Föderation auftreten. Die Koordinierung, die technischen Mittel und die Verpflegung führt die Föderationsleitung aus, die personellen Mittel stellen die nationalen Gesellschaften zur Verfügung. Die Föderation ist keine eigenständige juristische Persönlichkeit, sie wird auch nicht durch die völkerrechtlichen Konventionen und Abkommen geschützt, und sie ist kein Akteur des Völkerrechts. Die Vereinten Nationen haben die Eigenständigkeit der Föderation nicht anerkannt, obwohl die Föderation bis heute darum kämpft, die gleiche Stellung wie das ICRC zu bekommen. Die IFRC behauptet sich jedoch über die Rechte und Pflichten der international anerkannten ICRC. Dies ist auch nicht weiter problematisch, da alle nationalen Gesellschaften der ICRC unterliegen und durch sie ihren humanitären völkerrechtlichen Schutz bekommen, dadurch also subsidiär auch die IFRC.

Die IFRC besitzt keinen eigenen Sitz (da jeder Sitz der nationalen Organisationen zugleich auch als Sitz der Föderation dienen kann), jedoch hat sie eine teils selbstständige innere Struktur und Organe, letztendlich (auch wenn das von den Organen der Föderation nicht immer so gesehen wird) hat jedoch die endgültige Entscheidungsgewalt das Internationale Komitee.

Die Föderation umfasst in Friedenszeiten da jede nationale Gesellschaft mit allen Freiwilligen, die ihr angehört, insgesamt 105 Millionen Mitglieder (Ehrenamtliche, Kinder und Jugendliche des Jugendrotkreuzes und andere Helfer). Zu den 105 Millionen Mitgliedern gehören auch die Mitglieder der Jugendrotkreuz-Bewegungen. Diese Bewegungen werden durch das Anstreben der Föderation seit 1920 gegründet. Sie fungieren als Teil der nationalen Gesellschaften.

\footnotetext{
${ }^{24}$ HANTOS, A Magyar Vöröskereszt 100 éve 32.
} 
Diese Jugendorganisationen der Föderation ermöglichen somit, dass sich nicht nur Erwachsene dem Roten Kreuz anschließen können, sondern auch schon Schüler ab dem ersten Schuljahr. Neben den 105 Millionen Freiwilligen und Ehrenamtlichen Mitgliedern umfasst die Föderation und das Komitee zusammen insgesamt auch noch 300.000 Hauptamtliche Mitarbeiter (bezahlte Angestellte) in 185 nationalen Gesellschaften (Stand 2006). Man kann also gut erkennen, dass die Bewegung in erster Linie auf das Ehrenamt (unbezahlte Freiwillige) baut, und nur wenige Hauptamtliche (bezahlte Angestellten) hat.

Des Weiteren hält die Föderation z.Z. ca. 100 strategisch wichtige sogenannte Hilfsstützpunkte aufrecht. Falls auf der Welt sich eine Natur- oder der gleichzustellenden andere Katastrophe ereignet, kann die Liga seine Tätigkeit mit Unterstützung des am nächstliegenden Hilfsstützpunktes aufnehmen. ${ }^{25}$

\section{Zusammenfassung}

Wenn wir nun einen fiktiven Spaziergang durch die Geschichte der internationalen RotkreuzBewegung machen würden, wären die Sehenswürdigkeiten die Schlacht von Solferino im Jahre 1859, die Dunant dazu verleitet hat, seine Eindrücke der Weltregierung in seinem Buch Eine Erinnerung an Solferino darzulegen. Anhand dieses Buches ist unser nächster Halt die Gründung des Internationalen Komitees der Hilfsgesellschaften für Verwundetenpflege, zudem die Entstehung der heute als ICRC bekannten internationalen Organisation am 17. Februar 1863. Die Gründung des Komitees führt uns nun in das Jahr 1869, wo wir Zeuge eines der wichtigsten völkerrechtlichen und rechtsgeschichtlichen Momente des humanitären Völkerrechts werden. Näher die Ratifizierung der I. Genfer Konvention am 8. August 1869 und später die Verabschiedung der II., III. und IV. Genfer Konventionen. Den Weg weiterführend kommen wir in das Jahr 1919, wo neben der völkerrechtlich anerkannten internationalen Organisation, die auf die im Vorfeld gegründeten nationalen Gesellschaften basierende IFRC, also die Föderation gegründet wurde. Dieser letzte Akt führte dazu, dass ab dem Jahre 1919 die Bewegung zwei internationale Organisationen und mehrere nationale Gesellschaften verzeichnen kann. Zusammenfassend kann man sagen, dass aus dem Gedanken von Dunant die größte humanitäre Hilfsorganisation der Welt zustande kam, die ihre Aufgaben auf drei strukturell teils unabhängige Säulen basierend ausübt. Diese Säulen sind die nationalen Gesellschaften, das ICRC und die IFRC.

25 MVK-OI, A vöröskereszt története eszmeterjesztőknek 105-125. 


\section{Literatur- und Quellenverzeichnis}

I. Genfer Konvention $=$ Genfer Abkommen vom 12. August 1949 zur Verbesserung des Loses der Verwundeten und Kranken der bewaffneten Kräfte im Felde (GA I) https://www.swiss-archives.ch/detail.aspx?ID=29355732 (25. 12. 2018)

II. Genfer Konvention = Abkommen vom 12. August 1949 zur Verbesserung des Loses der Verwundeten, Kranken und Schiffbrüchigen der bewaffneten Kräfte zur See (GA II)

https://www.swiss-archives.ch/detail.aspx?ID=29355732 (25. 12. 2018)

III. Genfer Konvention = Abkommen über die Behandlung der Kriegsgefangene

https://www.ris.bka.gv.at/GeltendeFassung.wxe?Abfrage=Bundesnormen\&Gesetzesnummer=10000191 (25. 12. 2018)

IV. Genfer Konvention $=$ Abkommen über den Schutz von Zivilpersonen in Kriegszeiten https://www.admin.ch/opc/de/classified-compilation/19490188/index.html (25. 12. 2018)

Protokoll I und Protokoll II = Zusatzprotokolle vom 8. Juni 1977 zu den Genfer Abkommen vom 12. August 1949 https://www.swiss-archives.ch/detail.aspx?ID=29356589 (25. 12. 2018)

Protokoll III = Zusatzprotokoll vom 8. Dezember 2005 zu den Genfer Abkommen vom 12. August 1949 über die Annahme eines zusätzlichen Schutzzeichens

https://www.swiss-archives.ch/detail.aspx?ID=6424329 (25. 12. 2018)

BOISSIER, Pierre: History of the International Committee of the Red Cross. Volume I: From Solferino to Tsushima.

Geneva 1985

BoIssier, Pierre: Henry Dunant. Revue internationale de la Croix-Rouge, Aug. 1974, 443-464

Boppe, Roger: L'homme et la guerre. Le Docteur Louis Appia et les débuts de la Croix-Rouge. Geneva 1959

FIRPO, L. (ed.): Henri Dunant e le origini della Croce Rossa. Roma 1979

FRANÇOIS, A.: Un grand humanitaire. Revue internationale de la Croix-Rouge, März 1928, 203-244.

MOOREHEAD, Caroline: Dunant's dream: War, Switzerland and the history of the Red Cross. London 1998

Deutsches Rotes Kreuz: Das Rote Kreuz und seine Grundsätze. https://www.drk.de/das-drk/geschichte/wissenund-helfen/das-rote-kreuz/die-sieben-rotkreuzgrundsaetze/ (25. 12. 2018)

DUNANT, Jean Henri: Solferinoi emlékek. Budapest 1978

DUNANT, Jean Henri: Eine Erinnerung an Solferino. Eigenverlag des Österreichischen Roten Kreuzes. Wien 1997

DuRAnD, André: Gustave Moynier and the peace societies. International Review of the Red Cross, 314/1996 532-

550 .

DurAnD, André: The first Nobel Prize (1901) Henry Dunant, Gustave Moynier and the International Committee of the Red Cross as candidates. International Review of the Red Cross, 842/2001 275-285.

DuRAND, Roger: Théodore M. est aussi un fondateur de la Croix-Rouge. Gesnerus 34/1977 139-155

DuRAND, Roger: Henry Dunant und die Ostschweiz. Geneva 1992

DuRAnD, Roger (ed.): De l'utopie à la réalité. Actes du Colloque Henry-Dunant. tenu à Genève au palais de l'Athénée et à la chapelle de l'Oratoire les 3, 4 et 5 mai 1985 = Collection Henry Dunant No 3. Genève 1988

HANTOS János: A Magyar Vöröskereszt 100 éve. Budapest 1981

HeudTLAss, Willy: J. Henry Dunant, Gründer des Roten Kreuzes, Urheber der Genfer Konvention. Eine Biographie in Dokumente. Kohlhammer Verlag 1962

MOLNÁR Viktor: A genfi conventio. Budapest 1887

Magyar Vöröskereszt Országos Irodája (MVK-OI): Magyar Szent Korona Országai Vơrôs-Kereszt Egyletének története. Budapest 1904

Magyar Vöröskereszt Országos Irodája (MVK-OI): A vöröskereszt története eszmeterjesztőknek. Msc. Budapest 2010 PETERS, Tom F.: Transitions in Engineering: Guillaume Henri Dufour and the Early 19th Century Cable Suspension Bridges. Birkhauser 1987 